\title{
Is the routine pressure dressing after thyroidectomy necessary? A prospective randomized controlled study
}

\author{
Patorn Piromchai ${ }^{1}$, Patravoot Vatanasapt ${ }^{1}$, Wisoot Reechaipichitkul ${ }^{1}$, \\ Warinthorn Phuttharak ${ }^{2}$ and Sanguansak Thanaviratananich*1
}

\author{
Address: ${ }^{1}$ Department of Otorhinolaryngology, Faculty of Medicine, Khon Kaen University, Thailand and ${ }^{2}$ Department of Radiology, Faculty of \\ Medicine, Khon Kaen University, Thailand \\ Email: Patorn Piromchai - patorn@gmail.com; Patravoot Vatanasapt - patravoot@gmail.com; Wisoot Reechaipichitkul - wisree@gmail.com; \\ Warinthorn Phuttharak - koi_www@yahoo.com; Sanguansak Thanaviratananich* - sthanaviratananich@gmail.com \\ * Corresponding author
}

Published: 20 March 2008

BMC Ear, Nose and Throat Disorders 2008, 8:1 doi:10.1186/1472-68/5-8-1
Received: 13 February 2008

Accepted: 20 March 2008

This article is available from: http://www.biomedcentral.com/l472-68/5/8/I

(C) 2008 Piromchai et al; licensee BioMed Central Ltd.

This is an Open Access article distributed under the terms of the Creative Commons Attribution License (http://creativecommons.org/licenses/by/2.0), which permits unrestricted use, distribution, and reproduction in any medium, provided the original work is properly cited.

\begin{abstract}
Background: An acute complication of thyroidectomy is fatal hematoma, which can produce an upper airway obstruction needing immediate intubation or tracheostomy. After neck surgery, we usually apply a pressure dressing with a non-woven, adhesive fabric to reduce bleeding and fluid collection at the operative bed. We conducted a prospective, randomized, controlled study to evaluate a pressure vs. a non-pressure dressing after thyroid surgery by monitoring blood and serum in the operative bed.
\end{abstract}

Methods: We studied 108 patients who underwent II 6 thyroid surgeries at Srinagarind Hospital, Khon Kaen University, between December 2006 and September 2007. The patients were randomized to either the pressure dressing or non-pressure dressing group. Ultrasound of the neck was performed $24 \pm 3$ hours after surgery. The volume of fluid collection in the operative bed was calculated. All patients were observed for any post-operative respiratory distress, wound complications, tingling sensation or tetany.

Results: The distributions of age, sex, surgical indications and approaches were similar between the two groups. There was no statistically significant difference in the volume of fluid collection in the operative bed $(p=0.150)$ and the collected drained content $(p=0.798)$. The average time a drain was retained was 3 days. One patient in the pressure dressing group suffered cutaneous bruising while one patient in the non-pressure dressing group developed immediate hemorrhage after the skin sutures.

Conclusion: Pressure dressing after thyroidectomy does not have any significant impact on decreasing fluid collection at the operative bed. The use of pressure dressing after thyroidectomy may not therefore be justified.

Trial Registration: NCT00400465, ISRCTN52660978 


\section{Background}

About 200 cases of thyroidectomy are performed per year at Srinagarind Hospital, Khon Kaen University. An acute complication of this operation can lead to a fatal hematoma due to an upper airway obstruction (in 1 to 2.5 percent of cases) [1-4], requiring either intubation or tracheostomy.

After surgery, we usually apply a pressure dressing using a non-woven, adhesive, fabric to help reduce bleeding and collection of fluid on the basis that it may help to decrease dead space, compress bleeding vessels, decrease the slipping of ligature, the re-opening of cauterized veins, and the oozing area, all of which might precipitate a hematoma at the operative bed. The obvious external evidence of hematoma is a change in the neck contour or bulging skin flaps $[5,6]$. These signs may be obscured by a widely closed area of the pressure dressing and may delay detection of the hematoma, leading to airway obstruction.

We conducted a prospective, randomized, controlled study to assess the role of a pressure $v s$. non-pressure dressing after thyroid surgery, by monitoring fluid accumulation in the operative bed by ultrasound and by collecting serum in suction drains.

\section{Methods}

Approval was sought from the Khon Kaen University Ethics Committee for Human Research before initiating the study and informed consent was given by the patients. The study was carried out on 108 patients who underwent 116 thyroid surgeries at Srinagarind Hospital, Khon Kaen University, between December 2006 and September 2007. The discrepancy between the number of patients and the number of surgeries was due to 8 patients having undergone completion thyroidectomies for well-differentiated thyroid carcinomas confirmed by histopathology. These 8 patients were randomized as fresh cases with no consideration given to any previous surgery.

We excluded patients (1) with cervical lymph node metastases requiring neck dissection, (2) clinical or laboratory indicators of coagulation disorders or (3) whose bleeding cannot be stopped by ligation and/or cautery or (4) with anaplastic (undifferentiated) carcinoma, which is typically large and invades adjacent structures.

We stratified patients into two groups according to operation field extension. The first group comprised patients who underwent lobectomy or completion thyroidectomy and the second group patients who underwent subtotal or total thyroidectomy. The patients in each group were then randomized to either the pressure or non-pressure dressing group using a computer-generated randomization list with balanced blocks of unequal size. The treatment assignments were contained in sealed, opaque, sequentially-numbered, individual envelopes. After randomization, a nurse, not involved in the surgery, opened the envelope and independently prepared the dressing material. We used conventional knot-tying ligation for medium-sized vessels and the monopolar electrothermal vessel-sealing system for small-sized vessels to achieve vascular control. The operating surgeon was informed of the assignment group after having completed closure of the wound to avoid bias during operation. In the pressure dressing group, gauze was placed throughout the operative area and pressure applied with a non-woven, adhesive fabric $\left(\right.$ Hypafix $\left.^{\circledast}\right)$; in the non-pressure dressing group, gauze was placed on the incision site and held in place with permeable plastic tape (Transpore ${ }^{\circledR}$ ).

The closed suction drain, with negative pressure (Radi$\operatorname{vac}^{\circledR}$ ), was threaded through a separate wound. A single drain was used in patients who underwent lobectomy or completion thyroidectomy $v$ s. two closed suction drains for patients who underwent subtotal or total thyroidectomy.

Volume ultrasound (GE Logiq ${ }^{\circledR}$ ) of the neck was performed after surgery $24 \pm 3$ hours by the same head and neck radiologist or under his/her supervision. The dressing material was removed at the ward before the patient was taken for ultrasound so as not to apprise the radiologist of the type of dressing. The volume of fluid collection in the operative bed was calculated by measuring the maximum diameter in three dimensions; on the assumption that collection would be in the form of an ellipse or sphere [7]. The volume of fluid collected (in the suction drain) was measured separately. Drains were removed when the fluid collection fell below $10 \mathrm{~mL} / \mathrm{day}$. All patients were observed for any post-operative respiratory distress, wound complication, tingling sensation or tetany. The specimens were subjected to histopathological examination for final confirmation of diagnosis. Patients were discharged after drain removal.

The sample-size was calculated based on preliminary data obtained in one month. The 5 patients who underwent thyroidectomy were sent to ultrasound. The mean of fluid volume in surgical bed was $1.53 \mathrm{~mL}$ and the standard deviation was $2.5 \mathrm{~mL}$. A sample size of 116 patients was calculated to be able to detect a difference volume of 1.6 $\mathrm{mL}$ with a two-sided test with a type 1 error of $5 \%$ with a power of $90 \%$ and adjusted $5 \%$ for dropouts.

Statistical analyses were conducted using Stata (version 9, StataCorp LP, Texas, USA). The normal distributions of data were tested by Kolmogorov-Smirnov test. The effectiveness between the pressure and non-pressure dressing groups were analyzed using the unpaired $t$ test or Mann- 
Whitney U test for continuous variables $v$ s. the Pearson's $\chi^{2}$-test for categorical variables as appropriate. For all tests, $p<0.05$ was considered statistically significant.

\section{Results}

Of the 116 thyroid surgeries conducted, there were 15 $(12.9 \%)$ males and $101(87.1 \%)$ females. The average age of patients was 45.45 years (range, 15-76). Pressure dressings were used for 57 patients and non-pressure dressings for 59. A protocol violation occurred in 2 patients assigned to the pressure dressing group as the surgeon used only one drain instead of two after a subtotal thyroidectomy.

Of the operations performed $65.5 \%$ (in 76 patients) were lobectomy. Age and sex were statistically similar between the two groups. The reasons for surgery included: thyroid nodule in $65(56 \%)$ patients, multinodular goiter in 23 $(19.8 \%)$, Graves' disease in $9(7.8 \%)$ and thyroid carcinoma in $19(16.4 \%)$. The distributions of the surgical indications and approaches were similar between the two groups (Table 1).

On day one post-surgery, the amount of fluid collection in the operative bed was assessed for both groups using ultrasound. The average volume in the pressure vs. nonpressure dressing groups was 0.195 and $0.408 \mathrm{~mL}$, respectively. The $t$-test was used to assess for any significant difference in the average amount of fluid collected between the two groups and there was no statistically significant difference in the volume of fluid collected in the operative bed $(p=0.150)$. The average drained content from the pressure $v s$. the non-pressure dressing groups was 59.88 and $62.63 \mathrm{~mL}$, respectively, which was not a statistically significant difference $(p=0.798)$ (Table 2).
The average time a drain was retained was 3 days and the average hospital stay was 5 days. There was no statistically significant difference between groups $(p=0.225$ and 0.543 , respectively). One patient in the pressure dressing group suffered cutaneous bruising; while an immediate hemorrhage after skin suture developed in one patient from the non-pressure dressing group. In the latter case, we re-explored and found ligation material was dislodged from anterior jugular vein. No patient had any post-operative respiratory distress, tingling sensation or tetany (Table 3).

\section{Discussion}

Hematoma is a rare complication of thyroid surgery but the condition can cause an upper airway obstruction, albeit rare (range, 1 to 2.5 percent). The etiology includes slipping of ligature on major vessels (which occurred in this study), reopening of cauterized veins, retching, vomiting, bucking during recovery, valsalva maneuver, increased blood pressure during recovery, large dead space and oozing from the cut area of the thyroid [6]. In our institute, the surgeon usually applies a pressure dressing on the basis that it may help to decrease dead space, compress bleeding vessel and limit the oozing area. Furthermore, it prevents the patient from moving the neck freely which may help to decrease slippage of the ligature or reopening of cauterized veins.

Although many surgeons have gone beyond abandoning a pressure dressing to completely avoiding the use of a drain $[8,9]$, to our knowledge there has been no prospective randomized study to prove the benefits of pressure dressing in post-thyroidectomy patients. We designed our study to use ultrasonography of the neck to measure fluid collection in the thyroid bed to provide an objective assessment of a method commonly used in many reports $[10,11]$. The weakness of our study was that we did not

Table I: Demographics, diagnoses and types of thyroid surgery performed

\begin{tabular}{lcc}
\hline & Pressure dressing $(n=57)$ & Non-pressure dressing $(n=59)$ \\
\hline Demographics & & 44.10 \\
Age & 46.39 & $51(86.4)$ \\
Sex (female) (\%) & $50(87.7)$ & $30(50.8)$ \\
Diagnoses & $35(61.4)$ & $11(18.6)$ \\
$\quad$ Thyroid nodule (\%) & $12(21.1)$ & $5(8.5)$ \\
Multinodular goiter (\%) & $4(7.0)$ & $13(22.0)$ \\
Graves' disease (\%) & $6(10.5)$ & 51 \\
Well-differentiated thyroid carcinoma (\%) & 47 & 38 \\
Types of thyroid surgery & 42 & 13 \\
Group I & 5 & 8 \\
Lobectomy & 10 & 1 \\
Completion thyroidectomy & 4 & 7 \\
Group 2 & 6 &
\end{tabular}


Table 2: Volume of fluid in the operative bed and the volume of drained fluid

\begin{tabular}{|c|c|c|c|c|}
\hline & Pressure dressing $(n=57)$ & Non-pressure dressing $(n=59)$ & $p$-value & $\begin{array}{l}\text { 95\% Confidence Interval of the } \\
\text { difference }\end{array}$ \\
\hline $\begin{array}{l}\text { Volume of fluid in operative bed } \\
(\mathrm{mL})\end{array}$ & 0.1954 & 0.4083 & $0.150^{a}$ & $-0.69-0.26^{a}$ \\
\hline \multicolumn{5}{|l|}{ Volume of drains fluid } \\
\hline Day I (mL) & 52.07 & 53.98 & $0.156^{a}$ & $-10.01-6.18 \mathrm{a}$ \\
\hline Day $2(\mathrm{~mL})$ & 7.28 & 7.03 & $0.877^{a}$ & $-3.75-4.24^{a}$ \\
\hline Day $3(\mathrm{~mL})$ & 0.53 & 1.36 & $0.087^{a}$ & $-2.73-1.07^{a}$ \\
\hline Total $(\mathrm{mL})$ & 59.88 & 62.63 & $0.798^{a}$ & $-13.82-8.32^{a}$ \\
\hline
\end{tabular}

$P<0.05$ was considered significant

a Calculated with unpaired $t$-test

have an objective control of pressure on the skin after applying the pressure or non-pressure dressing. This problem was discussed among the investigators before initiating the study, and we decided not to use the pressure meter because we wanted to make the study approximate regular practice.

Our average hospital stay was slightly longer (5 days) than that of other institutions (3-6 days)[12,13], perhaps due to the variable definition of hospitalization and the criteria for discharging patients in each study. We admitted patients 1 day prior to surgery and we counted the day we discharged the patient (usually in the morning) as one day. In uncomplicated cases, the patients were discharged from the hospital after drain removal.

In our study, when using a pressure dressing over against a non-pressure dressing, fluid collection at the operative bed and collected drained content was lower in the pressure dressing group. This matched our hypothesis but there was no significant difference. Considering theory on the late detection of hematoma in pressure dressing due to a widely closed area, we did not find either immediate or late hematoma in this group. We suggest that the pressure dressing may have a benefit for preventing hematoma but a larger, randomized, controlled trial is required to establish this practice.

\section{Conclusion}

Pressure dressing after thyroidectomy does not provide any significant benefit vis-à-vis decreasing fluid collection at the operative bed. The use of a pressure dressing after thyroidectomy may, therefore, not be justified.

\section{Competing interests}

The author(s) declare that they have no competing interests.

\section{Authors' contributions}

PP designed and supervised the research; collected, analyzed, and interpreted the data; and drafted and revised the manuscript. PV contributed to the conceptual design of the study. WR contributed to the conceptual design and coordinated the project. WP did the ultrasonographic assessments. ST made substantial contributions to the design of the study and approved the final version of the manuscript. All authors read and approved the final manuscript.

\section{Acknowledgements}

The authors thank the staff and nurses at Srinagarind Hospital for their excellent care of the subjects enrolled in this study, Masayoshi Kobayashi, MD. for his advice and Mr. Bryan Roderick Hamman for assistance with the English-language presentation of the manuscript.

Table 3: Duration of retained drains, complications and length of hospital stay

\begin{tabular}{lcccc}
\hline & Pressure dressing $(n=57)$ & Non-pressure dressing $(n=59)$ & $p$-value & $\begin{array}{c}95 \% \text { Confidence Interval of the } \\
\text { difference }\end{array}$ \\
\hline $\begin{array}{l}\text { Duration of retained drains (days) } \\
\text { Complications }\end{array}$ & $2.32 \pm 0.51$ & $2.36 \pm 0.71$ & $0.225^{\mathrm{a}}$ & $-0.27-0.19 \mathrm{a}$ \\
$\quad$ Cutaneous bruising & 1 & 0 & $1.044^{\mathrm{b}}$ & \\
$\quad$ Hemorrhage & 0 & 1 & $0.975^{\mathrm{b}}$ & $0.543^{\mathrm{a}}$ \\
Hospital stay (days) & $4.37 \pm 0.52$ & $4.37 \pm 0.69$ & $-0.23-0.22^{\mathrm{a}}$ \\
\hline
\end{tabular}

$P<0.05$ was considered statistically significant

a Calculated using the unpaired $t$-test

${ }^{b}$ Calculated with the chi-square test 


\section{References}

I. Abbas G, Dubner S, Heller KS: Re-operation for bleeding after thyroidectomy and parathyroidectomy. Head Neck 200I, 23(7):544-546.

2. Agarwal A, Mishra SK: Post-thyroidectomy haemorrhage: an analysis of critical factors in successful management. J Indian Med Assoc 1997, 95(7):418-419, 433.

3. Blaivas $M$, Hom DB, Younger JG: Thyroid gland hematoma after blunt cervical trauma. Am J Emerg Med 1999, I 7(4):348-350.

4. Palestini N, Tulletti V, Cestino L, Durando R, Freddi M, Sisti G, Robecchi A: [Post-thyroidectomy cervical hematoma]. Minerva Chir 2005, 60(I):37-46.

5. Harding J, Sebag F, Sierra M, Palazzo FF, Henry JF: Thyroid surgery: postoperative hematoma - prevention and treatment. Langenbecks Arch Surg 2006, 39 I(3): I69-173.

6. Savargaonkar A: Post-Thyroidectomy Haematoma Causing Total Airway Obstruction - A Case Report. Indian J Anaesth 2004, 48(6):483-485.

7. Lee SJ, Ahn IM: Effectiveness of percutaneous ethanol injection therapy in benign nodular and cystic thyroid diseases: longterm follow-up experience. Endocr J 2005, 52(4):455-462.

8. Corsten $M$, Johnson $S$, Alherabi $A$ : Is suction drainage an effective means of preventing hematoma in thyroid surgery? A metaanalysis. J Otolaryngol 2005, 34(6):4I5-4I7.

9. Pothier DD: The use of drains following thyroid and parathyroid surgery: a meta-analysis. J Laryngol Otol 2005 I I 9(9):669-67I.

10. Khanna J, Mohil RS, Chintamani, Bhatnagar D, Mittal MK, Sahoo M Mehrotra $M$ : Is the routine drainage after surgery for thyroid necessary? A prospective randomized clinical study [ISRCTN63623 I 53]. BMC Surg 2005, 5: I I.

II. Schwarz W, Willy C, Ndjee C: [Gravity or suction drainage in thyroid surgery? Control of efficacy with ultrasound determination of residual hematoma]. Langenbecks Arch Chir 1996, 38 I(6):337-342

12. Ahluwalia S, Hannan SA, Mehrzad H, Crofton M, Tolley NS: A randomised controlled trial of routine suction drainage after elective thyroid and parathyroid surgery with ultrasound evaluation of fluid collection. Clin Otolaryngol 2007, 32(I):28-3I.

13. Terris DJ, Moister B, Seybt MW, Gourin CG, Chin E: Outpatient thyroid surgery is safe and desirable. Otolaryngol Head Neck Surg 2007, I 36(4):556-559.

\section{Pre-publication history}

The pre-publication history for this paper can be accessed here:

http://www.biomedcentral.com/1472-6815/8/1/prepub
Publish with Biomed Central and every scientist can read your work free of charge

"BioMed Central will be the most significant development for disseminating the results of biomedical research in our lifetime. "

Sir Paul Nurse, Cancer Research UK

Your research papers will be:

- available free of charge to the entire biomedical community

- peer reviewed and published immediately upon acceptance

- cited in PubMed and archived on PubMed Central

- yours - you keep the copyright
BioMedcentral 